\title{
Contribuições do Modelo Bioecológico à Análise do Desemprego sob a Perspectiva Familiar e de Gênero
}

Contribuciones del Modelo Bioecológico al Análisis del Desempleo en una Perspectiva Familiar y de Género

Contributions of Bioecological Model to the Unemployment Research from a Family and Gender

Perspective

Joyce Cristina dos Santos Aguiar

ORCID: https://orcid.org/0000-0002-5462-4914

Universidade do Porto, Porto/Portugal

\begin{abstract}
Declaração de Direito Autoral
A submissão de originais para este periódico implica na transferência, pelos autores, dos direitos de publicação impressa e digital. Os direitos autorais para os artigos publicados são do autor, com direitos do periódico sobre a primeira publicação. Os autores somente poderão utilizar os mesmos resultados em outras publicações indicando claramente este periódico como o meio da publicação original. Em virtude de sermos um periódico de acesso aberto, permite-se o uso gratuito dos artigos em aplicações educacionais e científicas desde que citada a fonte conforme a licença CC-BY da Creative Commons.
\end{abstract}

\section{Resumo}

O desemprego enquanto fenômeno psicológico torna-se, em tempos de recessão econômica, um objeto de estudo de particular interesse. Ampla é a literatura acerca das consequências negativas do desemprego sobre o bem-estar das pessoas nesta situação. No entanto, observa-se que um grande número dos estudos centra-se somente na pessoa em desemprego e em variáveis individuais (tais como idade, sexo, características de personalidade e duração do desemprego, por exemplo). O objetivo da presente revisão narrativa de literatura, foi apresentar como o modelo bioecológico pode contribuir para a análise do fenômeno do desemprego, conferindo complexidade e dinamismo na abordagem desta problemática. A pesquisa bibliográfica foi feita nas bases de dados Academic Search Complete, PsycArticles, PsycInfo, Scielo, Scopus e SocIndex. Considerando a família como uma teia complexa de relações sistêmicas, onde as interações se dão de forma bidirecional, compreende-se que seus diferentes subsistemas são afetados por pressões externas, mas também podem agir proativamente na preservação de um equilíbrio interno de funcionamento. Observou-se que o gênero é uma dimensão marcante para a articulação dos papéis familiares e profissionais, sendo esta uma variável que atravessa e condiciona a experiência de desemprego nas famílias heteronormativas com maior adesão aos papéis tradicionais de gênero. O presente trabalho integra parte da tese de doutorado da autora.

Palavras-chaves: Desemprego; Teoria bioecológica; Dinâmica familiar; Gênero.

\section{Resumen}

El desempleo como fenómeno psicológico se vuelve, en tiempos de recesión económica, un objeto de estudio de particular interés. Amplia es la literatura acerca de las consecuencias negativas del desempleo sobre el bienestar de las personas en esta situación. Sin embargo, se observa que un gran número de estudios se centra sólo en la persona en desempleo y en variables individuales (tales como edad, sexo, características de personalidade y duración del desempleo, por ejemplo). El objetivo de la 
presente revisión narrativa de literatura fue presentar como el modelo bioecológico puede contribuir al análisis del fenómeno del desempleo, dando complejidad y dinamismo al abordaje de esta problemática. La búsqueda bibliográfica fue hecha en las bases Académico Search Complete, PsycArticles, PsycInfo, Scielo, Scopus y SocIndex. Considerando la familia como una red compleja de relaciones sistémicas, donde las interacciones se dan de forma bidireccional, se comprende que sus diferentes subsistemas son afectados por presiones externas, pero también pueden actuar proactivamente en la preservación de un equilibrio interno de funcionamiento. Se observó que el género es una dimensión marcada para la articulación de los papeles familiares y profesionales, siendo ésta una variable que atraviesa y condiciona la experiencia de desempleo en las familias heteronormativas con mayor adhesión a los papeles tradicionales de género. Este artículo es parte de la tesis doctoral del autor.

Palabras claves: Desempleo; Teoria bioecológico; Dinámica familiar; Género.

\begin{abstract}
Unemployment as a psychological phenomenon becomes, in times of economic recession, an object of study of particular interest. Ample is the literature about the negative consequences of unemployment on the well-being of people in this situation. However, it is observed that a large number of studies focus only on the unemployed person, and as well on individual variables (such as age, sex, personality characteristics, and duration of unemployment, for example). This narrative literature review aims to present how the bioecological model can contribute to the analysis of the unemployment phenomenon, conferring it complexity and dynamism in the approach to this problem. The bibliographic search was performed on Academic Search Complete, PsycArticles, PsycInfo, Scielo, Scopus and SocIndex databases. Considering the family as a complex web of systemic relations, where interactions take place in a bidirectional way, their different subsystems are affected by external pressures, but they can also act proactively in the preservation of an internal balance of functioning. It was observed that gender is a significant dimension for the articulation between family and professional roles, being this a variable that crosses and conditions the unemployment experience in heteronormative families with greater adherence to traditional gender roles. This paper is part of the doctoral thesis of the author.
\end{abstract}

Keywords: Unemployment; Bioecological theory; Family dynamics; Gender.

\section{Introdução}

O fenômeno do desemprego, originalmente abordado sob uma perspetiva político-econômica, foi ao longo das décadas ampliando as suas fronteiras de conhecimento, tornando-se objeto de estudo também de outras disciplinas, dentre elas a Psicologia. De uma forma geral, o desemprego costuma ser categorizado de acordo com duas dimensões: a) normativa-institucional, para explicar o desemprego de modo objetivo e formal, sob o enfoque da estrutura econômica; e b) biográfica-subjetiva, que aborda as repercussões psicossociais do fenômeno do desemprego, como, por exemplo, a atribuição de estereótipos ao desempregado ou a instabilidade subjetiva que é refletida nas relações interpessoais (Ribeiro, 2010). Em tempos de recessão econômica, a insegurança oriunda do cenário de instabilidade econômica, (i.e, incertezas relacionadas com a perda do emprego, nomeadamente incertezas sobre conseguir ou não honrar as despesas mensais familiares) gera um aumento dos problemas psicológicos, bem como um aumento do estresse no indivíduo que se estende ao agregado familiar (Heretick, 2013; Procter, Papadopoulos, \& McEvoy, 2010). São esses impactos de natureza psicossocial do desemprego que se tornam o enfoque das Ciências Sociais (Coelho-Lima, Costa, \& Bendassolli, 2013). 
Os primeiros estudos com um olhar biográfico-subjetivo acerca dos efeitos psicológicos decorrentes da situação de desemprego tiveram início após a Grande Depressão de 1929, com o clássico trabalho de Jahoda, Lazarsfeld e Zeisel (ver Jahoda, Lazarsfeld, \& Zeisel, 2003). Esse trabalho empírico na pequena comunidade de Marienthal acerca do impacto do encerramento das atividades fabris na vida dos trabalhadores e da comunidade foi o ponto de partida para a elaboração de um modelo teórico - o modelo funcionalista ou "teoria da privação. Segundo Jahoda (1982), a situação de desemprego priva o indivíduo de benefícios que são oriundos de um emprego. Tais benefícios podem ser manifestos ou latentes. Por benefícios manifestos, entende-se aqueles que são tangíveis, como a remuneração que permite o acesso a bens materiais e concretos. Por benefícios latentes, estariam aqueles que não são palpáveis mas contribuem para o pleno funcionamento psicológico do indivíduo. De seguida, serão abordados brevemente cada um dos cinco benefícios apontados por Jahoda (1982) como os mais relevantes.

O primeiro benefício latente do qual a pessoa em desemprego está privada é a estruturação do tempo. Nas sociedades pósindustriais, as pessoas estão habituadas a uma rotina, que lhes foi incutida desde criança com os horários da escola, e segue para o âmbito profissional, por meio das jornadas de trabalho. A depender do tipo de trabalho que exerce (desde os mais "braçais", técnicos ou altamente qualificados), a rotina pode ser mais ou menos flexível e tem a função de estruturar as demais atividades que fazem parte de sua vida. Quando essa estrutura desaparece, quer seja de um modo planejado (e.g. aposentadoria) ou mais inesperadamente (e.g. demissão involuntária), a sua ausência geralmente origina sentimento de vazio, inutilidade, aborrecimento e ociosidade. De acordo com o que Jahoda (1982) observou na comunidade de Marienthal, até mesmo os finsde-semana haviam perdido, para os trabalhadores em desemprego, o seu significado culturalmente imposto.

Uma outra componente limitada pela situação de desemprego seria a da vinculação de metas individuais a metas coletivas. De acordo com a autora, para além da família nuclear, é no âmbito das organizações de trabalho que se encontra mais fortemente o contexto da valorização da coletividade, presentes quer na forma em que as tarefas estão divididas entre os empregados, quer no compartilhamento informal das experiências cotidianas (Jahoda, 1982). Esta componente encontra-se bastante interligada ao contato social. Mesmo nas pessoas mais tímidas ou nas quais o emprego não exige grande contato social, há uma "ampliação do conhecimento da realidade social ao observar as semelhanças e diferenças entre seus próprios costumes, experiências e opiniões e os das pessoas que estão ao seu redor" (Jahoda, 1982, p. 45). Afastado da demonstração cotidiana de coletividade, expressa no contato diário por meio da atividade de trabalho (i.e., com os colegas de trabalho, clientes ou fornecedores), o indivíduo poderá sofrer perda de um sentido de finalidade comum, exclusão de um grupo e isolamento social. No entanto, a intensidade de tais sentimentos poderá depender tanto de características do indivíduo quanto do contexto em que se insere. A autora argumenta que, em alguns casos (e.g., falência da empresa), poderá ainda haver o compartilhamento de experiências e sentimentos entre o grupo, mesmo sem o contato no ambiente de trabalho. De destacar que a redução do contato social é vista como uma privação não porque o indivíduo deixa de ter relações sociais, mas sim porque estas que são derivadas do emprego complementam-se com as demais redes sociais do indivíduo (família extensa e amigos). Além disso, a carga emocional das interações sociais no trabalho é diferente daquela dentro de uma família, que são mais intensas e emocionalmente mais fortes. Em contrapartida, as relações no ambiente laboral geralmente são mais do tipo racional do que emocional, ou seja, são oportunidades para 
adquirir maior conteúdo informativo, valoração sobre diferentes opiniões dos colegas, conhecimento de costumes e modos de vida das pessoas.

O quarto benefício do qual a pessoa em desemprego estaria privada engloba dois conceitos - estatuto e identidade. Por estatuto, a autora refere-se à valoração social que depende do contexto cultural, enquanto a identidade seria uma imagem que a pessoa tem de si. É consensual pela cultura atribuir aos diferentes postos de trabalho um estatuto social, que pode constituir como um dos elementos da identidade pessoal. Dessa maneira, a autora presume que para aquelas pessoas que antes ocupavam um posto de trabalho hierarquicamente superior, a situação de desemprego poderá ter um impacto negativo maior devido à perda de estatuto, repercutindo na imagem que a pessoa faz de si mesma.

Um último ponto de privação referido por Jahoda é o da atividade regular, sendo este muito relacionado à natureza da atividade de trabalho antes exercida. Isto porque, para alguns trabalhadores, o desemprego significa muitas vezes o rompimento com as atividades laborais (e.g., um maquinista não consegue continuar a desempenhar a sua atividade laboral após o despedimento, ao passo em que um professor pode, mesmo desempregado, exercer atividades autônomas como dar aulas particulares ou organizar cursos).

O modelo funcionalista de Jahoda recebeu, no entanto, algumas críticas. Para Fryer (1986), este modelo não seria suficiente para explicar o impacto negativo do desemprego sobre o indivíduo. Embora reconheça a importância dos benefícios latentes para a saúde mental, a principal crítica apontada seria a de que esta abordagem centra-se sobretudo na privação psicológica, colocando os indivíduos numa condição de passividade face às forças externas. Uma outra crítica apontada ao modelo funcionalista é a de que sua construção foi feita num contexto e período específico, não se podendo generalizar as conclusões para sociedades menos industrializadas ou para os dias atuais. Não obstante, o trabalho de Jahoda foi pioneiro na contribuição da compreensão do impacto psicológico do desemprego e até hoje é referência para muitos estudos nesta área (Sage, 2017).

Recorrendo ao modelo da agência pessoal, Fryer (1986) defende que o ser humano é um ser ativo e dinâmico e que, durante a situação de desemprego, torna-se limitado nas suas capacidades de tomada de decisão, resolução de problemas e realização de atividades. Em outras palavras, o principal efeito negativo de uma situação de desemprego seria a inibição da agência pessoal que é peculiar ao humano, interferindo em outras áreas da vida adulta (e.g., ter de adiar a decisão de casar ou de ter filhos por não ter um emprego). Tal inibição seria sobretudo devido ao impacto econômico do desemprego, que limitaria o acesso a outros recursos importantes, tais como atividades sociais, comida, casa e segurança física (Fryer, 1997). Assim, a queda no rendimento condiciona a agência pessoal, pois limita a capacidade da pessoa de planejar e organizar estilos de vida satisfatórios, impactando negativamente o seu bem-estar (Fryer, 1997). No seguimento a este modelo explicativo, vários são os estudos que têm vindo a constatar maior tensão e menor bem-estar em pessoas em desemprego (Aguiar, Matias, \& Fontaine, 2017; Estramiana, Gondim, Luque, Lana, \& Dessen, 2012; Ferreira, Pedro, \& Francisco, 2015). Aliás, grande parte dos estudos acerca dos efeitos do desemprego a nível individual centra-se em questões da saúde, física ou mental. Os efeitos mais comumente reportados são, além das doenças gástricas e cardiovasculares (Eliason \& Storrie, 2009; Michaud, Crimmins, \& Hurd, 2016), o decréscimo de autoestima, aumento de ansiedade, de depressão, de uso de substâncias (e.g., álcool e cigarro) de probabilidade de suicídio (Herbig, Dragano, \& Angerer, 2013)

Com base no exposto, observa-se que a situação de desemprego tem importante 
impacto na vivência do indivíduo, tendo sua origem na perda direta do rendimento econômico porém adquire um significado psicológico e se estende também sobre aspetos psicossociais. Tal impacto dá-se tanto no foro individual (em termos de bem-estar, identidade e sentido de agência) como no âmbito das interações sociais. Observa-se que os modelos teóricos aqui apresentados, que são os modelos clássicos utilizados na análise psicossocial acerca do impacto do desemprego, centram-se sobretudo em aspectos individuais, não sendo suficientes para dar conta de uma análise sob a perspectiva familiar. Para colmatar essa lacuna, buscou-se, através de uma revisão narrativa de literatura, trazer o racional teórico do modelo bioecológico de Bronfenbrenner como uma lente que permitirá melhor compreender o fenômeno do desemprego no âmbito da dinâmica familiar.

\section{Método}

As revisões narrativas são sínteses qualitativas norteadas por uma questão abrangente e atravessadas pelo olhar do pesquisador, que a partir dos textos selecionados, estabelece relações, contextualiza e fornece novas perspetivas a uma determinada temática (Lawrence, 2011). Esse tipo de revisão costuma ser utilizado estrategicamente quando se objetiva descrever, de modo breve, o percurso do conhecimento até então, oferecendo um panorama geral sobre dada temática, o chamado "estado da arte". Nesse sentido, as revisões narrativas desempenham um importante papel na divulgação e avanço do conhecimento (Rother, 2007). A revisão apresentada no presente estudo realizou-se no âmbito da tese doutoral da autora (ver Aguiar, 2017)

No presente estudo, realizou-se a pesquisa bibliográfica nas bases de dados Academic Search Complete, PsycArticles, PsycInfo, Scielo, Scopus e SocIndex. No entanto, como recomendado para sínteses narrativas (Aoki, Enticott, \& Phillips, 2013), utilizou-se também outras fontes (nomeadamente, livros clássicos da área) de modo a tornar mais diversificada e crítica a análise qualitativa, estando ela marcada profundamente pela ótica do pesquisador que "narra" a revisão. A busca foi feita em 2017, nos idiomas português, inglês, francês e espanhol. Os textos recuperados serão apresentados, em forma de síntese qualitativa, na secção a seguir.

\section{Resultados}

De modo a facilitar a leitura, a narrativa foi dividida em duas partes: a primeira aborda de que modo as esferas familiar e profissional (denominadas por "mesosistema trabalho-família") podem ser lidas à luz do modelo bioecológico e, a segunda, aborda como o gênero encontra-se associado ao desempenho dos papéis familiares e profissionais.

\section{O mesosistema trabalho-família}

A abordagem bioecológica, desenvolvida e reformulada ao longo dos anos por Bronfenbrenner e seus colaboradores (1979/1996), considera que quatro importantes aspetos encontram-se inter-relacionados: pessoa, processo, contexto e tempo. De acordo com Bronfenbrenner e Morris (1998, p. 995) o desenvolvimento seria o processo referente à "estabilidade e mudanças nas características biopsicológicas dos seres humanos ao longo do curso de suas vidas e através das gerações". Os autores ressaltam a importância das relações interpessoais no desenvolvimento e o conceito de reciprocidade, considerando que, numa díade, se um dos elementos passa por um processo de desenvolvimento, automaticamente estará contribuindo para o desenvolvimento do outro elemento, pela via dos processos proximais. Por meio dos processos proximais, as pessoas estabelecem relações em diferentes níveis e a sua participação ativa em interações permite o desenvolvimento intelectual, emocional, moral e social, sendo esta interação fortemente influenciada pelo contexto no qual se manifesta. De acordo com essa teoria, o 
ambiente no qual se dá o desenvolvimento humano deve ser compreendido topologicamente como "uma organização de estruturas concêntricas, estando cada uma contida na seguinte" (Bronfenbrenner, 1996 p. 18). Cada uma das estruturas é chamada pelo autor de: micro, meso, exo e macrossistema.

Um microssistema seria o ambiente no qual a pessoa participa ativamente e está envolvida em interações face-a-face, com características físicas e materiais especificas. Assim, a família, o local de trabalho e a escola seriam por exemplo microssistemas, nos quais há um padrão de atividades, papéis e relações interpessoais que são experienciadas pela pessoa e reforçadas pelo meio. O mesosistema refere-se às interações entre dois ou mais sistemas nos quais a mesma pessoa participa ativamente e que sobredetermina o tipo de interação que desenvolve (e.g., a relação que uma mãe, que trabalha numa empresa específica, tem com o seu local de trabalho e com a sua família).

O exossistema seria um sistema no qual, embora a pessoa não participe ativamente, interfere imediatamente no seu microssistema (e.g. para uma criança seria o local de trabalho da mãe, pois embora a criança não participe ativamente desse sistema, os horários de trabalho da mãe, por exemplo, interferem na disponibilidade da mesma no seu ambiente familiar). Por fim, o macrossistema seria o sistema que abrange todos os outros sistemas, formando interconexões que se diferenciam de cultura para cultura. Portanto, seria o sistema de valores e crenças que permeiam as diversas culturas e são assimilados pelas pessoas no processo de desenvolvimento.

Em relação ao desemprego de pessoas casadas e com filhos, a família é um microssistema de fundamental importância, no qual cada membro do agregado está em constante interação com outros microssistemas; além disso, está inserida num macrossistema e, portanto, dependente das condições econômicas e socioculturais deste. A perspectiva sistêmica considera que $\mathrm{o}$ sistema familiar é composto por subsistemas que se inter-relacionam, a saber: individual, conjugal, parental e fraternal. Cada membro do agregado familiar atua em diferentes subsistemas, desempenhando a cada nível um determinado papel. No subsistema individual, considera-se cada indivíduo como um microssistema, complexificando assim a teia de relações que se estabelecem entre os membros do agregado familiar. No subsistema conjugal, os cônjuges que atuam neste subsistema deverão ser capazes de estabelecer fronteiras entre este e os outros subsistemas, de modo a protegê-lo de interferências externas (a família de origem de cada um dos cônjuges, por exemplo) e manter o bom funcionamento interno. Com a presença de uma primeira criança no seio familiar, deverá também ser capaz de diferenciar o subsistema conjugal do parental, sendo que neste último o papel a desempenhar será de cuidado e educação, além de socialização do novo membro do agregado. Finalmente, com o surgimento de mais um filho, surgirá também mais um subsistema familiar, no qual os integrantes deverão ser capazes de interagir entre si, desenvolvendo competências de interação entre pares, cooperação e competição (Relvas, 1996). Portanto, a unidade familiar é um sistema composto por indivíduos, cujos comportamentos afetam e são afetados quer pelos comportamentos dos outros, quer pelos subsistemas nos quais estão inseridos, conferindo-lhe complexidade e dinamismo.

Desse modo, o sistema familiar é constantemente desafiado a, ao mesmo tempo em que recebe as pressões externas, encontrar um equilíbrio interno para o seu funcionamento. Para Relvas (1996), o sistema familiar possui limites que atuam como membranas semipermeáveis, permitindo a passagem seletiva de informações em ambas as direções: da família para o meio e do meio para os subsistemas familiares. Quem "controla" o grau de abertura desses limites são os membros da família, nomeadamente o casal, por meio das normas. Em outras palavras, a família não está passivamente 
sujeita às pressões externas, embora não lhe seja imune. Antes, atua por meio de um dinamismo próprio em busca de consistência e equilíbrio entre as forças internas e externas (Relvas, 1996).

Pelo exposto acima, entende-se que a situação de desemprego vivida por um dos elementos de uma família poderá repercutir nos demais membros do agregado, por diferentes vias. Por exemplo, interações no próprio microssistema familiar afetarão todos os membros; além disso, considerando que este microssistema está inserido num macrossistema cultural, os valores dessa cultura e em particular as expectativas ligadas aos papéis de gênero e ao exercício dos papéis profissional e familiar poderão constituir-se ameaças externas à dinâmica familiar, mas também ameaças internas pois estas permeiam as próprias normas familiares (pressões internas).

\section{Gênero e os papéis profissionais e familiares}

As teorias acerca das razões da diferenciação entre homens e mulheres face ao trabalho começaram a ganhar força a partir da segunda metade do século XIX. As primeiras tentaram justificar a posição dos dois sexos por predisposições biológicas (Auspurg, Iacovou, \& Nicoletti, 2017). Subjacente a esse pensamento da "natureza feminina", estava o conceito burguês de família, que alicerçado na ideia da inferioridade e fragilidade da mulher, colocava-a como responsável pela casa e afastava-a do trabalho remunerado (Martinengo, Jacob, \& Hill, 2010). São solidificadas assim as imagens do homemoperário e da mulher-doméstica, atribuindo ao homem o desempenho do papel instrumentalprovedor e, à mulher, o papel expressivocuidador (Parsons, 1964).

A partir do movimento feminista, a temática da diferenciação entre os sexos passa a ser estudada também pela psicologia, sendo introduzidos os sentimentos, comportamentos e atitudes que seriam típicos do "ser homem" ou "ser mulher" (Nitsche \& Grunow, 2016). A partir dos anos 80 do século passado, a perspectiva situacional permite à psicologia social compreender que, em vez da polarização entre o biológico, o social e o psicológico, é a interação entre essas componentes em um dado contexto que desenvolve e define o gênero e suas influências sobre o indivíduo. (Nitsche \& Grunow, 2016). Dessa forma, é preciso considerar o caráter dinâmico, situacional e relacional do gênero (Vieira, 2006).

Sem desconsiderar as pressões sofridas ao longo do processo de socialização da criança nem os processos biológicos, a teoria do papel social postula que as diferenças de gênero estão associadas aos diferentes papéis sociais desempenhados por homens e mulheres e às inferências das características psicológicas associadas a cada papel (Eagly \& Wood, 2013). Tais expectativas, geradas no grupo e pelo grupo aos quais pertencem, são a base dos estereótipos e refletem as diferenças na divisão sexual do trabalho. De acordo com Deaux e Major (1987) a exibição em maior ou menor grau dos comportamentos típicos do homem ou da mulher depende: a) das expectativas dos observadores, ou seja estereótipos, atitudes e representações sobre o que é ser homem e ser mulher; b) de concepções pessoais, que agem como "profecias de realização automática" uma vez que o indivíduo assimila as expectativas dos espectadores como suas e atua conforme o que lhe é esperado; c) de fatores situacionais, ou seja, as crenças diferenciais sobre homens e mulheres podem ser transmitidas mais claramente ou de modo mais sutil conforme a cultura e normas sociais.

$\begin{gathered}\text { Assim, a classificação dos } \\ \text { indivíduos como }\end{gathered}$
comportamentos dos comunal" (comunal) ou "agêntico" (agentic)
varia em função do papel que desempenham.
Em outras palavras, as pessoas atuam de modo
mais agêntico (i.e., expressão de liderança,
determinação e participação social) e menos
comunal (i.e., empenhados no cuidado para o
bem-estar dos outros, nomeadamente da
família e do lar) quando são colocadas em um


papel dominante, passando de um tipo de comportamento a outro em função da sua posição na situação de interação. Em um estudo realizado por Eagly e Steffen (ver Eagly \& Wood, 2013), observou-se, por um lado, que a crença que as pessoas tradicionalmente atribuem à mulher (mais dócil, amável, carinhosa e compreensiva do que o homem) não se mantém quando se pensa numa mulher trabalhadora. Por outro lado, os homens que desempenhavam tarefas domésticas eram vistos como mais sensíveis nas relações interpessoais. Nesse sentido, não seriam observadas diferenças entre características predominantes nos homens e mulheres se, a ambos, fosse dada a oportunidade de se distribuírem de modo equivalente pelos diversos papéis sociais.

A partir do final da década de 80 , com a influência dos movimentos feministas e dos estudos de Derrida e Foucault, surgem as teorias de construção do gênero, que buscam compreender como as relações de gênero se dão a partir das interações sociais e discursivas de poder, privilegiando assim uma abordagem construtivista (Hirata, 2014). Nesse sentido, pode-se dizer que o gênero possui dimensões relacionais e dinâmicas, sendo constantemente construído pelas vivências nos mais diversos domínios de vida, dentre eles o laboral e o familiar, pelo que alguns autores referem o doing gender (West \& Zimmerman,1987).

Não obstante às mudanças na sociedade contemporânea no que diz respeito a igualdade entre homens e mulheres, ainda é possível reconhecer papéis sociais diferenciados ou largamente específicos ao homem e à mulher. Para Vieira (2006) "de todos os papéis que até à atualidade menos mudaram para a mulher, encontram-se o de esposa e de mãe" (p. 77). Embora a tradicional divisão do homem como ganha-pão e da mulher como responsável pela vida doméstica esteja a diminuir, é fato que existe uma segregação sexual a nível das profissões, estando com maior frequência as mulheres a ocupar cargos que requerem "qualidades femininas" e os homens em trabalhos que exigem "qualidades masculinas" (Vieira, 2006), estes geralmente com posições hierarquicamente superiores e mais bem remunerados.

Também na esfera familiar, essa ocupação de diferentes papéis mantem-se. De acordo com um estudo acerca da igualdade de género em Portugal, que utilizou uma amostra representativa da população, relativamente à execução do trabalho doméstico, o homem permanece como um ajudante secundário da mulher (Wall et al., 2016). O referido estudo reporta que atualmente os homens, independentemente da faixa etária, participam mais do trabalho doméstico, no entanto esta participação traduz-se ainda mais numa decisão de colaborar com a vida familiar do que na assunção plena de um papel familiar.

De fato, alguns estudiosos postulam a divisão tradicional do trabalho profissional e familiar como uma forma de preservar a identidade de gênero masculina e feminina (Nitsche \& Grunow, 2016; West \& Zimmerman, 1987). Assim, poderia ser explicado o fato de alguns homens financeiramente dependentes de suas esposas realizarem ainda menos atividades domésticas do que os que não estão sob essa condição (Aguiar, Matias, Barham \& Fontaine, 2018). Efetivamente, uma vez que não estavam exercendo sua função de provedor, que confirmaria socialmente a sua identidade masculina, tentariam sobretudo afastar-se da função feminina de cuidadora do lar para não serem considerados "menos masculinos". Assim, ao executar o trabalho doméstico, as mulheres demonstram a sua feminilidade, a si próprias e aos outros, enquanto ao não executar o trabalho familiar, os homens afirmam a sua masculinidade (Davis \& Greenstein, 2013; Poeschl, 2010).

Parece assim que, não obstante as várias mudanças na sociedade oriundas do ingresso e permanência feminina no mercado de trabalho, ainda é muito presente a figura do gênero masculino como provedor da família. Em virtude disso, poderá ser ainda mais difícil para homens com atitudes de gênero mais 
tradicionais conseguir lidar com a tensão gerada pela situação de desemprego, que poderá ser encarada como uma ameaça ao seu papel de provedor, potenciando assim o efeito negativo da experiência de desemprego. Para as mulheres com maior adesão aos papéis sociais tradicionais, no entanto, estar responsável pelo bem-estar da família poderá minimizar esse impacto negativo. Embora também experienciem a tensão e as consequências psicossociais advindas da situação de crise, o investimento no papel tradicional de cuidadora poderá desempenhar um efeito protetor sobre a sua própria experiência de desemprego (Knabe, Schöb, \& Weimann, 2015).

Assim, considerando o papel do gênero e das expectativas face ao desempenho profissional e familiar, é importante distinguir a situação de desemprego quando esta é protagonizada pelo homem ou pela mulher, uma vez que estas configurações poderão ter repercussões distintas conforme o modo como os elementos do casal lidam com tais expectativas sociais.

\section{Considerações finais}

O presente estudo insere-se no âmbito da tese doutoral da autora. Neste recorte, buscou-se, através de uma revisão de literatura, trazer um contributo que permitisse um olhar mais amplo acerca do desemprego enquanto fenômeno que ultrapassa o foro individual. Para isso, utilizou-se como referencial a teoria bioecológica do desenvolvimento humano, de Bronfenbrenner. Utilizou-se também a perspectiva do gênero de modo a contextualizar as implicações do desemprego quer no âmbito da esfera profissional, quer no âmbito das relações conjugais e familiares.

Apesar do desemprego ser, em primeira instância, oriundo do mercado econômico e financeiro, é possível fazer várias leituras acerca deste fenômeno, sob diferentes prismas, tais como o da Economia, Sociologia, Direito do Trabalho e da Psicologia. Ampla é a literatura acerca das consequências negativas do desemprego sobre o bem-estar das pessoas nesta situação, nomeadamente, diminuição de felicidade, autoestima e satisfação com a vida (Estramiana et al, 2012; Haid \& SeiffgeKrenke, 2013), aumento de sintomas como estresse, ansiedade e depressão (Procter et al., 2010), impactando na saúde geral e aumentando o risco de distúrbios psicológicos e suicídio (Santos, 2015). No entanto, observase que um grande número dos estudos se centra somente na pessoa em desemprego e em variáveis individuais (idade, sexo, características de personalidade, duração do desemprego etc.). Com menor frequência são os estudos em que se analisam as suas consequências no âmbito da dinâmica familiar. Assim, o presente estudo ancorou-se no modelo bioecológico para mostrar de que forma esse racional teórico poderá contribuir para a análise do desemprego de um modo mais sistêmico.

Para além da privação de recursos materiais, faz-se pertinente refletir sobre o impacto do desemprego na dinâmica familiar, considerando os três subsistemas familiares (i.e., individual, conjugal e parental). Em outras palavras, tendo o desemprego tamanhas consequências sobre o indivíduo, é preciso considerar também de que modo tais repercussões se refletem na sua rede próxima de relações interpessoais, sobretudo nas interações da família nuclear, de modo a melhor sustentar não só as intervenções clínicas decorrentes da consequência psicológica do desemprego mas também fornecer contribuições a políticas públicas face a este fenômeno.

Por fim, analisar o desemprego no âmbito das dinâmicas familiares é também considerar o papel do gênero como diferenciador da experiência. Faz-se importante referir que tal diferenciação, no entanto, não se relaciona a características biológicas associadas ao sexo, mas são sim resultantes da configuração dos papéis sociais atribuídos ao homem e à mulher nas nossas sociedades. Não obstante as mudanças na sociedade contemporânea no que diz respeito 
a igualdade entre homens e mulheres, ainda é possível reconhecer papéis sociais exclusivos ou largamente específicos ao homem e à mulher. Embora a tradicional divisão do homem como ganha-pão e da mulher como responsável pela vida doméstica e bem-estar dos membros da família esteja a extinguir-se, é fato que existe uma clara diferenciação sexual na perceção das características dos homens e das mulheres, que se reflete no âmbito profissional. Assim, em contextos heteronormativos, o sexo do cônjuge em desemprego poderá ser um condicionante da forma como a família lidará com a experiência de desemprego. Ora, sendo uma experiência, deve-se ter sempre em atenção as idiossincrasias e peculiaridades, não se pretendendo, portanto, generalizar ou enquadrá-las na mesma ordem.

\section{Referências}

Aguiar, J. (2017). O papel do gênero na experiência do desemprego de casais portugueses e brasileiros. Tese de Doutorado não-publicada. Faculdade de Psicologia e Ciências da Educação da Universidade do Porto, Portugal.

Aguiar, J., Matias, M., Barham, E. J., \& Fontaine, A. M. (2018). Efeitos do desemprego sobre o casal: Uma revisão sistemática. Gerais: Revista Interinstitucional de Psicologia, 11(1), 174-189.

Aguiar, J., Matias, M., \& Fontaine, A. M. (2017). Desemprego, satisfação com a vida e satisfação conjugal em portugueses e brasileiros. Revista Psicologia: Organizações e Trabalho, 17(4), 210-217. doi: 10.17652/rpot/2017.4.13751

Aoki, N. J., Enticott, J. C. \& Phillips, L. E. (2013). Searching the literature: Four simple steps. Transfusion, 53(1), 14-17. doi: 10.1111/j.1537-2995.2012.03790.x

Auspurg, K., Iacovou, M. \& Nicoletti, C. (2017). Housework share between partners: Experimental evidence on gender-specific preferences. Social Science Research, 66, 118-139.

Bronfenbrenner, U. (1996). A ecologia do desenvolvimento humano: Experimentos naturais e planejados. Porto Alegre: Artes Médicas

Bronfenbrenner, U., \& Morris, P. A. (1998). The ecology of developmental processes. In W. Damon (Ed.), Handbook of child psychology (5th ed., Vol. 1, pp. 993-1028). New York: Wiley
Coelho-Lima, F.,Costa, A. L. F. \& Bendassolli, P. F. (2013). A produção científica da Psicologia brasileira acerca do desemprego. Universitas Psychologica, 12(4), 1283-1299. doi: 10.11144/Javeriana.UPSY12-4.pcpb.

Davis, S. N., \& Greenstein, T. N. (2013). Why study housework? Cleaning as a window into power in couples. Journal of Family Theory \& Review, 5(2), 63-71. doi; 10.1111/jftr.12004

Deaux, K., \& Major, B. (1987). Putting gender into context: An interactive model of gender-related behavior. Psychological Review, 94, 369-389. doi: 10.1037/0033295X.94.3.369.

Eagly, A. H., \& Wood, W. (2013). The naturenurture debates: 25 years of challenges in understanding the psychology of gender. Perspectives on Psychological Science, 8(3), 340-357.

Eliason, M. \& Storrie, D. (2009). Job loss is bad for your health - Swedish evidence on cause-specific hospitalization following involuntary job loss. Social Science \& Medicine, 68(8), 1396-1406. doi: 10.1016/j.socscimed.2009.01.021

Estramiana, J. L. A., Gondim, S. M. G., Luque, A. G., Luna, A. F. \& Dessen, M. C. (2012). Desempleo y bienestar psicológico en Brasil y España: Un estudio comparativo. Revista Psicologia: Organizações e Trabalho, 12(1), 5-16. Recuperado de http://submissionpepsic.scielo.br/index.php/rpot/index 
Ferreira, S., Pedro, M. F. \& Francisco, R. (2015). Entre marido e mulher, a crise mete a colher: A relação entre pressão económica, conflito e satisfação conjugal. Psicologia, 29(1), 11-22. Recuperado de http://www.scielo.mec.pt/scielo.php?script $=$ sci $\operatorname{arttext} \& \mathrm{pid}=\mathrm{S} 0874-$ $20492015000100002 \& \operatorname{lng}=$ pt\&tlng=pt.

Fryer, D. (1986). Employment deprivation and personal agency during unemployment: A critical discussion of Jahoda's explanation of the psychological effects of unemployment. Social Behaviour, 1, 3-23. Recuperado de http://psycnet.apa.org/record/1989-24290$\underline{001}$

Fryer, D. (1997). Agency restriction. In N. Nicholson (Ed.), The Blackwell encyclopedic dictionary of organizational psychology. Oxford, England: Blackwell.

Haid, M-L. \& Seiffge-Krenke, I. (2013). Effects of (un)employment on young couples' health and life satisfaction. Psychology and Health, 28(3), 284-301. doi: 10.1080/08870446.2012.720983.

Herbig, B., Dragano, N. \& Angerer, P. (2013). Health in long-term unemployed.

Deutsches Arzteblatt International, 110(2324), 413-419. doi: 10.3238/arztebl.2013.0413

Heretick, D. (2013). Clinicians' reports of the impact of the 2008 financial crisis on mental health clients. Journal of Social, Behavioral, and Health Sciences, 7(1), 121. doi: 10.5590/JSBHS.2013.07.1.01

Hirata, H. (2014). Gênero, classe e raça: Interseccionalidade e consubstancialidade das relações sociais. Tempo Social, 26(1), 61-73.

Jahoda, M. (1982). Employment and unemployment. A socio-psychological analysis. Cambridge: Press of University of Cambridge.

Jahoda, M., Lazarsfeld, P., \& Zeisel, H. (2003). Marienthal: The sociography of an unemployed community. New Brunswick, Transaction Publisher.

Knabe, A., Schöb, R. \& Weimann, J. (2015). Partnership, gender, and the well-being cost of unemployment. Social Indicators
Research, 124(2), 1-21. doi: 10.1007/s11205-015-1167-3.

Lawrence, C. N. (2011). Writing a literature review in social sciences. Recuperado de https://www.academia.edu/2911352/Writin g_a_Literature_Review_in_the_Social_Sci ences

Martinengo, G., Jacob, J. I. \& Hill, E. J. (2010). Gender and the Work-Family Interface: Exploring Differences Across the Family Life Course. Journal of Family Issues, 31(10), 1363-1390. doi: 10.1177/0192513x10361709

Michaud, P.C., Crimmins, E. M. \& Hurd, M. D. (2016). The effect of job loss on health: Evidence from biomarkers. Labour Economics, 41, 194-203. doi: $\underline{10.1016 / j . l a b e c o .2016 .05 .014}$

Nitsche, N. \& Grunow, D. (2016). Housework over the course of relationships: Gender ideology, resources, and the division of housework from a growth curve perspective. Advances in Life Course Research, 29, 80-94. doi: 10.1016/j.alcr.2016.02.001

Parsons, T. (1964). Family structure and the socialization of the child. In T. Parsons \& R. F. Balles (Eds.), Family, socialization and interaction process (pp. 35-133). London: Routledge \& Kegan Paul.

Poeschl, G. (2010). Desigualdades na divisão do trabalho familiar, sentimento de justiça e processos de comparação social. Análise Psicológica, 28(1), 29-42.

Procter, N., Papadopoulos, I., \& McEvoy, M. (2010). Global economic crises and mental health. Advances in Mental Health, 9, 210214.

Relvas, A. P. (1996). O ciclo vital da família: Perspectiva sistémica. Porto: Edições Afrontamento.

Ribeiro, M. A. (2010). Estratégias micropolíticas para lidar com o desemprego: Contribuições da psicologia social do trabalho. Psicologia Política, 9(18), 331-346.

Rother, E. T. (2007). Revisão sistemática x revisão narrativa. Editorial. Acta Paulista de Enfermagem, 20(2). doi: $\underline{10.1590 / \mathrm{S} 0103-21002007000200001}$ 
Sage, D. (2017). Reversing the negative experience of unemployment: A mediating role for social policies? Social Policy Administration, 52(5), 1043-1059. doi: $\underline{10.1111 / \mathrm{spol} .12333}$

Santos, M. (2015). Unemployment, mental health worker and suicide: A systematic review. European Psychiatry, 30(1), 28-31. doi: $10.1016 / \mathrm{S} 0924-9338(15) 30295-9$
Vieira, C. M. C. (2006). É menino ou menina? Género e educação em contexto familiar. Coimbra: Almedina.

Wall, K., Cunha, V., Atalaia, S., Rodrigues, L., Correia, R. V. \& Rosa, R. (2016). Livro Branco. Homens e igualdade de género em Portugal. Lisboa: Instituto de Ciências Sociais.

West, C., \& Zimmerman, D. H. (1987). Doing gender. Gender and Society 2(1), 125-

151.doi: $10.1177 / 0891243287001002002$.

\section{Dados sobre os autores:}

- Joyce Cristina dos Santos Aguiar: Psicóloga pela Universidade Federal do Ceará, Brasil, e doutora em Psicologia pela Universidade do Porto, Portugal, em regime de dupla titulação com a Universidade Federal de São Carlos, Brasil. Atualmente, atua como pesquisadora postdoc na Escola de Engenharia da Universidade do Minho, Portugal. 